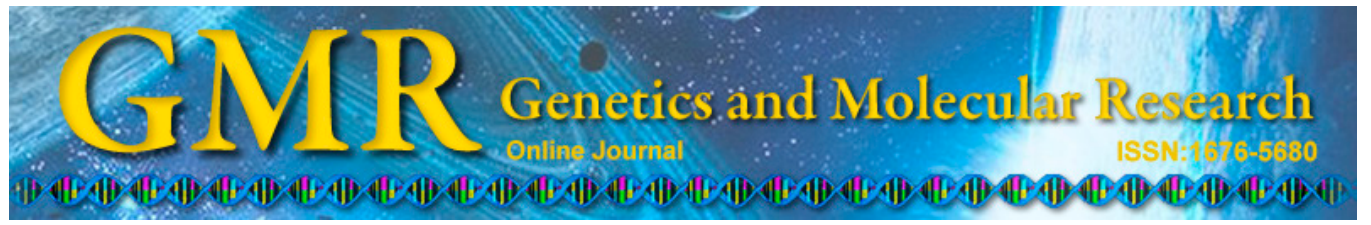

\title{
QTLs for rice flag leaf traits in doubled haploid populations in different environments
}

\author{
J. Cai ${ }^{1}$, M. Zhang ${ }^{1}$, L.B. Guo ${ }^{1}$, X.M. Li ${ }^{1}$, J.S. Bao ${ }^{2}$ and L.Y. Ma ${ }^{1}$ \\ ${ }^{1}$ State Key Laboratory of Rice Biology, \\ China National Rice Research Institute, Hangzhou, China \\ ${ }^{2}$ Institute of Nuclear Agricultural Sciences, Zhejiang University, \\ Hangzhou, China \\ Corresponding authors: J.S. Bao / L.Y. Ma \\ E-mail: jsbao@zju.edu.cn / ricemaly@163.com
}

Genet. Mol. Res. 14 (2): 6786-6795 (2015)

Received September 4, 2014

Accepted February 13, 2015

Published June 18, 2015

DOI http://dx.doi.org/10.4238/2015.June.18.21

\begin{abstract}
Two rice doubled haploid (DH) populations derived from the crosses of ZYQ8/JX17 and CJ06/TN1 were used to detect quantitative trait loci (QTLs) for flag leaf length (FLL), width (FLW), and angle (FLA) under long-day conditions in Hangzhou (subtropical zone) and short-day conditions in Hainan (tropical zone), China. The four parents differed significantly in all 3 traits. FLL was found to be positively correlated with FLW in the 2 populations. A total of 30 QTLs were identified for flag leaf traits, with a contribution to the phenotypic variation of each QTL from 4.49 to $26.30 \%$. Among these, $q F L L-4 b$, $q F L W-12$, and $q F L A-2 a$ showed larger additive effects on the phenotype and explained more variations compared to the other QTLs. $q F L L-1 a$ and $q F L L-8$ were detected in both environments, while $q F L L-2, q F L L-$ $3, q F L L-10, q F L L-12, q F L W 11, q F L W 2$, and $q F L A 8$ were novel QTLs, which may be beneficial to rice ideal-type breeding.
\end{abstract}

Key words: Doubled haploid population; Quantitative trait loci; Flag leaf; Rice 


\section{INTRODUCTION}

Rice flag leaf is the main photosynthetic organ after heading, which provides approximately $40 \%$ of the photosynthetic products for grain formation. The flag-leaf contributes to $44-48 \%$ yield in an individual plant, of which $40 \%$ contributes to grain setting rate and $10 \%$ to 1000-grain weight (Tang, 1998; Zhang et al., 2001; Yang and Wang, 2001). Flag leaf shape is also an important component of ideal plant type in rice, and flag leaf length (FLL), width (FLW), and angle (FLA) are vital selection indices for rice breeding (Pan et al., 2004).

Leaf morphology is controlled by genetic factors, and genetic studies of rice flag leaf shape, FLL, and FLW indicate that they are quantitative traits that are primarily controlled by polygenes (Lu et al., 1979; Ling et al., 2008) or controlled by 1 or 2 pairs of genes (Shen, 1983; Yan and Wang, 1990; Lu et al., 2005). Quantitative trait locus (QTL) mapping has been successfully applied for identifying genes/QTLs important in controlling leaf morphology. In previous studies, Li et al. $(1998,1999)$ studied Lemont/Teqing F2:4 populations and identified 3 QTLs related to FLL and FLW and 5 QTLs controlling FLA. Li et al. (2000), using the JX17/ ZYQ8 DH population, detected 2 QTLs - $q F L L 4$ and $q F L L 8$ for FLL, 4 QTLs for FLW, and 2 QTLs for flag leaf areas. Among these QTLs, 1 QTL on chromosome 8 controlling length, width, and flag leaf area was at the same location. Since then, QTL mapping for flag leaf traits has been extensively conducted in different types of genetic populations, such as near isogenic lines, backcross inbred lines, doubled haploids (DHs), and $\mathrm{F}_{2}$, with more than 100 QTLs identified (Dong et al., 2003; Kobayashi et al., 2003; Shen et al., 2003; Wang et al., 2004; Yue et al., 2006; Cao et al., 2007; Tong et al., 2007; Farooq et al., 2010; Ding et al., 2011; Wang et al., 2011; Sonah et al., 2012; Chen et al., 2012). For example, Kobayashi et al. (2003) mapped QTLs for FLL, FLW, and FLA using the Milyang23/Akihikari RIL population over 5 seasons, and found a total of 9 genomic regions involved in leaf development. Peng et al. (2007) analyzed QTLs for flag leaf traits using 2 different populations, the JX17/ZYQ8 DH population and Zhengshan97/Minghui63 RIL population, and found that the QTLs for leaf length and areas were the same as those reported by $\mathrm{Li}(2000)$.

Although a number of QTLs for rice flag leaf traits have been detected, few have been subjected to fine mapping and none has been cloned. Wang et al. (2011) narrowed a QTL for flag leaf size (leaf length, width, and area) on chromosome 1 to a 31-kb region containing 4 predicted genes. Ding et al. (2011) finely mapped a QTL to chromosome 4 controlling root volume per tiller and co-segregating with FLW and spikelet number per panicle to a $38-\mathrm{kb}$ region containing 3 open reading frames, including the previously characterized NARROW LEAF 1 (NAL1) gene. Similarly, Chen et al. (2012) finely mapped a major QTL for FLW qFLW4 to a 74.8-kb interval that also contained the gene NAL1. In addition, mutants for leaf size, including narrow leaf 7 (Fujino et al., 2008), NAL1 (Qi et al., 2008), and narrow and rolled leaf 1 (Hu et al., 2010), have been isolated by mutant analysis and map-based cloning. These mutants are not useful for crop improvement, while the stable, reliable, and repeatedly expressed QTLs are useful for marker-assisted selection to improve the plant architecture and yield potential. However, QTLs are easily influenced by genetic background and environment (Peng et al., 2007), and thus it is necessary to search for QTLs in different populations under different environmental conditions.

In this study, 2 rice DH populations were planted in 2 environments to map the QTLs for flag leaf traits. Our objective was to identify reliable markers or genomic regions that can be used for marker-assisted selection to improve the ideal plant type of rice. 


\section{MATERIAL AND METHODS}

\section{Plant materials}

Two DH populations derived from 2 crosses between an indica rice ZYQ8 and a japonica rice JX17 and a japonica rice CJ06 and an indica rice TN1, respectively, were used in this study. The ZYQ8/JX17 population consists of 127 lines, and a genetic linkage map including 234 restriction fragment length polymorphism and simple sequence repeat markers has been constructed previously (Li et al., 2002; Dong et al., 2003). The CJ06/TN1 population consists of 120 lines and a genetic linkage map with 211 simple sequence repeat markers, which was constructed previously (Zhang et al., 2006; Ma et al., 2009b).

\section{Investigation of flag leaf traits}

Field trials were conducted in 2 environments. The first was carried out during the winter season (short-day conditions) in Linshui, Hainan Province (HN, $\left.18^{\circ} 30^{\prime} \mathrm{N}, 110^{\circ} 02^{\prime} \mathrm{E}\right)$, China during late 2007 and early 2008. The second experiment was carried out in a normal (long-day conditions) environment at the farm of the China National Rice Research Institute, Hangzhou (HZ, $\left.30^{\circ} 04^{\prime} \mathrm{N}, 119^{\circ} 55^{\prime} \mathrm{E}\right)$, China, in 2008 . The environmental conditions from May through October in Hangzhou differ dramatically from the conditions from November through March in Hainan (Bao et al., 2004). The experiment design was a completely randomized block with 2 replications. In each replication, all DH lines and 4 parents were transplanted and arranged randomly in the field into 4 rows with 8 plants per row for each line. The plant-to-plant spacing between and within rows was $20 \mathrm{~cm}$. The flag leaf traits, including FLL, FLW, and FLA, were measured at 10 days after flowering of the main stem panicle. The middle 5 plants in the middle 2 rows of each DH line (10 plants per line) were investigated for each accession in each replication.

\section{Correlation analysis}

Determination of the correlation between FLL, FLW, and FLA was carried out using the SAS program 9.1 (SAS Institute Inc., Cary, NC, USA).

\section{QTL analysis}

Interval QTL mapping was conducted using the Mapmarker/QTL software version 1.1 to analyze the QTLs for flag leaf-related traits. A minimum log 10-likelihood ratio (LOD) score of 2.0 was considered to indicate the presence of a putative QTL in a given genomic region, and the variation and additive effect of each QTL was also calculated. QTL nomenclature followed that described by McCouch et al. (1997).

\section{RESULTS}

\section{Performance of flag leaf-related traits in parents and DH population}

FLL, FLW, and FLA of the 4 parents and 2 DH populations are shown in Table 1. In the ZYQ8/JX17 population, all traits showed a significant difference between the 2 parents in $\mathrm{HN}$, 
but no difference in HZ, except for FLA. In the CJ06/TN1 population, TN1 showed a significant longer flag leaf than CJ06 in HZ, but no difference in HN was observed. The FLA of the 2 parents showed a significant difference in both environments, but the opposite effects of the environments were found; in HZ, TN1 had a larger FLA than CJ06, whereas in HN, TN1 had a smaller FLA than CJ06. The results clearly indicated that flag leaf traits were greatly affected by the environment.

All 3 traits showed wide segregation among the DH lines (Table 1), and the average of each trait was close to the mid-parent value. All 3 traits had a wider range of variation in $\mathrm{HZ}$ than in $\mathrm{HN}$ in both populations, except FLW in the ZYQ8/JX17 population. Continuous variation among the DH populations for each trait was observed with transgressive segregation in some DH lines, indicating the involvement of polygenic genes controlling these traits. Based on the data of skewness and kurtosis, both of FLL and FLW in the DH populations fit normal distributions, but FLA did not fit a normal distribution because the skewness and kurtosis were larger than 1 (Table 1).

\begin{tabular}{|c|c|c|c|c|c|c|c|c|}
\hline \multirow[t]{2}{*}{ Population } & \multirow[t]{2}{*}{ Traits } & \multirow[t]{2}{*}{ Environments } & \multicolumn{2}{|c|}{ Parents } & \multicolumn{4}{|c|}{ DH population } \\
\hline & & & & & Means \pm SD & Range & Skewness & Kurtosis \\
\hline \multirow[t]{7}{*}{ ZYQ8/JX17 } & & & ZYQ8 & JX17 & & & & \\
\hline & FLL & $\mathrm{HZ}$ & $25.32 \pm 4.33$ & $26.06 \pm 3.04$ & $30.38 \pm 6.68$ & $13.86-51.44$ & 0.517 & 0.367 \\
\hline & & $\mathrm{HN}$ & $16.52 \pm 2.57$ & $24.18 \pm 3.60^{* *}$ & $19.20 \pm 3.60$ & $10.24-27.39$ & 0.028 & -0.111 \\
\hline & FLW & $\mathrm{HZ}$ & $1.28 \pm 0.10$ & $1.23 \pm 0.09$ & $1.29 \pm 0.26$ & $0.77-2.14$ & 0.474 & 0.475 \\
\hline & & $\mathrm{HN}$ & $1.02 \pm 0.10$ & $1.23 \pm 0.13^{*}$ & $1.12 \pm 0.28$ & $0.74-2.49$ & 2.037 & 5.467 \\
\hline & FLA & $\mathrm{HZ}$ & $14.40 \pm 5.82$ & $21.90 \pm 4.43 * *$ & $19.59 \pm 8.28$ & $7.30-58.56$ & 1.520 & 3.700 \\
\hline & & $\mathrm{HN}$ & $11.40 \pm 5.13$ & $20.33 \pm 16.06^{* *}$ & $15.40 \pm 8.62$ & $4.20-53.50$ & 2.195 & 6.186 \\
\hline \multirow[t]{7}{*}{ CJ06/TN1 } & & & CJ06 & TN1 & & & & \\
\hline & FLL & $\mathrm{HZ}$ & $34.37 \pm 3.38$ & $38.27 \pm 3.92 *$ & $35.55 \pm 6.70$ & 21.64-53.35 & 0.270 & -0.290 \\
\hline & & $\mathrm{HN}$ & $20.37 \pm 3.59$ & $21.17 \pm 3.80$ & $21.18 \pm 4.44$ & $11.60-34.39$ & 0.402 & 0.411 \\
\hline & FLW & $\mathrm{HZ}$ & $1.58 \pm 0.04$ & $1.51 \pm 0.06$ & $1.54 \pm 0.20$ & $1.15 \pm 2.12$ & 0.396 & 0.264 \\
\hline & & $\mathrm{HN}$ & $1.38 \pm 0.11$ & $1.30 \pm 0.09$ & $1.34 \pm 0.15$ & $0.94-1.67$ & -0.150 & -0.526 \\
\hline & FLA & $\mathrm{HZ}$ & $11.30 \pm 1.06$ & $17.00 \pm 3.33^{* *}$ & $14.51 \pm 5.36$ & $5.80-39.60$ & 2.083 & 6.805 \\
\hline & & $\mathrm{HN}$ & $8.56 \pm 2.46$ & $6.18 \pm 2.04 *$ & $10.74 \pm 4.36$ & $5.60-33.70$ & 2.102 & 7.014 \\
\hline
\end{tabular}

$*$ and $* *$ indicated significant difference at $\mathrm{P}<0.05$ and 0.01 levels, respectively.

\section{Analysis of correlation}

Correlation coefficients among FLL, FLW, and FLA in the 2 DH populations are shown in Table 2. In the ZYQ8/JX17 population, FLL showed a positive correlation ( $\mathrm{r}=0.66$, $\mathrm{P}<0.01$ ) with FLW in only 1 environment $(\mathrm{HZ})$. In the CJ06/TN1 population, a significant correlation between FLL and FLW was observed in both environments.

Table 2. Correlation coefficients for flag leaf traits in two DH populations of different environments.

\begin{tabular}{llllr}
\hline Population & Trait & FLL & FLW & FLA \\
\hline ZYQ8/JX17 & FLL & & 0.1003 & -0.1646 \\
& FLW & $0.6619^{* *}$ & 0.1243 & 0.1487 \\
FLA & 0.1503 & $0.4522^{* *}$ & -0.0670 \\
CJ06/TN1 & & -0.1135 & 0.1504 \\
& FLL & $0.5045^{* *}$ & -0.0261 & \\
& FLW & FLA &
\end{tabular}

Above diagonal is for $\mathrm{HN}$ and below diagonal is for HZ. ${ }^{*}$ and **indicated significant difference at $\mathrm{P}<0.05$ and 0.01 levels, respectively. 


\section{QTL analysis}

The results of QTL analyses are shown in Table 3 and Figures 1 and 2. A total of 30 putative QTLs for 3 traits distributed on 11 chromosomes were detected in the $2 \mathrm{DH}$ populations. Seven QTLs for FLL were detected in the ZYQ8/JX17 population and 6 QTLs were detected in the CJ06/TN1 population, located on chromosomes 1, 2, 3, 4, 6, 8, 10, and 12. In the ZYQ8/JX17 population, the qFLL-1a flanked by the marker region R210-CT441 could be detected in 2 environments, explaining 6-7\% of the phenotypic variation. Four other QTLs on chromosome 2, 3, 4, and 12 for FLL were detected only in HZ, and another QTL on chromosome 10 was only detected in HN. In the CJ06/TN1 population, 1 QTL on chromosome 8 flanked by RM447 and RM3120 could be detected in both environments, explaining 11-16\% of the phenotypic variation. Two QTLs on chromosome 4 were detected in each environment. In HZ, 2 other QTLs on chromosomes 1 (qFLL-1b) and 6 (qFLL-6) were identified, accounting for approximately $20 \%$ of the phenotypic variation.

Table 3. QTL for flag leaf traits in two DH populations in different environments.

\begin{tabular}{|c|c|c|c|c|c|c|c|c|}
\hline Traits & DH population & Environments & Locus & $\mathrm{Chr}$ & Interval & LOD & Additive effect & Variance explained $\%$ \\
\hline \multirow[t]{13}{*}{ FLL } & \multirow[t]{7}{*}{ ZYQ8/JX17 } & \multirow[t]{5}{*}{$\mathrm{HZ}$} & $q F L L-1 a$ & 1 & R210-CT441 & 2.05 & 1.86 & 7.08 \\
\hline & & & $q F L L-2$ & 2 & G275-CT482 & 2.02 & -1.70 & 5.26 \\
\hline & & & $q F L L-3$ & 3 & G1318-RM148 & 2.05 & -1.52 & 5.12 \\
\hline & & & $q F L L-4 a$ & 4 & G177-CT206 & 4.43 & 2.54 & 13.58 \\
\hline & & & $q F L L-12$ & 12 & G2140-RG457 & 2.48 & 1.74 & 6.46 \\
\hline & & \multirow[t]{2}{*}{$\mathrm{HN}$} & $q F L L-1 a$ & 1 & R210-CT441 & 2.33 & 0.94 & 6.43 \\
\hline & & & $q F L L-10$ & 10 & G1125-G1084 & 3.25 & 1.10 & 8.46 \\
\hline & \multirow[t]{6}{*}{ CJ06/ TN1 } & \multirow[t]{4}{*}{$\mathrm{HZ}$} & $q F L L-1 b$ & 1 & RM428-RM5302 & 3.63 & 6.46 & 22.50 \\
\hline & & & $q F L L-4 b$ & 4 & RM252-RM3276 & 3.56 & -6.98 & 26.30 \\
\hline & & & $q F L L-6$ & 6 & RM340-RM494 & 3.74 & 6.16 & 19.90 \\
\hline & & & $q F L L-8$ & 8 & RM447-RM3120 & 2.98 & -5.73 & 16.90 \\
\hline & & \multirow[t]{2}{*}{$\mathrm{HN}$} & $q F L L-4 c$ & 4 & RM548-RM6997 & 2.27 & -4.31 & 23.80 \\
\hline & & & $q F L L-8$ & 8 & RM447-RM3120 & 2.11 & -3.01 & 11.20 \\
\hline \multirow[t]{8}{*}{ FLW } & \multirow[t]{5}{*}{ ZYQ8/JX17 } & \multirow[t]{4}{*}{$\mathrm{HZ}$} & $q F L W-3$ & 3 & C74A-RG450 & 2.68 & -0.07 & 6.65 \\
\hline & & & $q F L W-4 a$ & 4 & G177-CT206 & 6.11 & 0.11 & 18.44 \\
\hline & & & $q F L W-6$ & 6 & CT115-СТ506 & 3.83 & -0.09 & 9.98 \\
\hline & & & $q F L W-12$ & 12 & G148-RG413 & 6.72 & 0.12 & 20.85 \\
\hline & & $\mathrm{HN}$ & $q F L W-11$ & 11 & PTA818-RG2 & 2.59 & 0.08 & 8.13 \\
\hline & \multirow[t]{3}{*}{ CJ06/ TN1 } & \multirow[t]{2}{*}{$\mathrm{HZ}$} & $q F L W-1$ & 1 & RM428-RM5302 & 2.12 & 0.14 & 13.30 \\
\hline & & & $q F L W-4 b$ & 4 & RM3276-RM255 & 2.21 & -0.13 & 12.50 \\
\hline & & $\mathrm{HN}$ & $q F L W-2$ & 2 & RM145-RM521 & 3.08 & -0.13 & 17.00 \\
\hline \multirow[t]{9}{*}{ FLA } & \multirow[t]{9}{*}{ ZYQ8/JX17 } & \multirow[t]{3}{*}{$\mathrm{HZ}$} & $q F L A-6$ & 6 & CT506-G200 & 2.06 & -2.29 & 7.02 \\
\hline & & & $q F L A-8$ & 8 & CT195-G1073 & 2.01 & -2.12 & 5.90 \\
\hline & & & $q F L A-12 a$ & 12 & RZ397-G124-1A & 2.59 & -2.38 & 7.74 \\
\hline & & \multirow[t]{6}{*}{$\mathrm{HN}$} & $q F L A-1$ & 1 & C385-G370 & 4.60 & 3.15 & 12.50 \\
\hline & & & $q F L A-2 a$ & 2 & CT87-G1234 & 5.65 & 3.41 & 13.80 \\
\hline & & & $q F L A-2 b$ & 2 & G243A-GA43 & 4.01 & 2.76 & 9.70 \\
\hline & & & $q F L A-7$ & 7 & RG351-CT469 & 2.87 & -2.55 & 8.10 \\
\hline & & & $q F L A-9$ & 9 & CT100-RZ404 & 2.14 & -1.87 & 4.49 \\
\hline & & & $q F L A-12 b$ & 12 & G148-RG413 & 3.42 & -2.50 & 7.93 \\
\hline
\end{tabular}

In the ZYQ8/JX17 population, 4 QTLs on chromosomes 3, 4, 6, and 12 were detected for FLW in HZ, and 1 QTL on chromosome 11 was detected in HN, indicating that FLW was strongly affected by the environmental conditions. These QTLs explained 6.7\% (qFLW3) to $20.9 \%$ (qFLW-12) of the total phenotypic variation. qFLW-4a, flanked by G177 and CT206, was located at the same region as qFLL-4a. In the CJ06/TN1 population, 2 QTLs were detected in HZ, and 1 detected in HN for FLW. Similarly, no common QTL between the 
2 environments were detected. qFLW4b and qFLL-4b were located nearby on chromosome 4, sharing the same marker, RM3276 (Figure 2).

Nine QTLs, 6 in HZ and 3 in HN, were detected for FLA in the ZYQ8/JX17 population, but no QTL was detected in the CJ06/TN1 population. These QTLs had a relatively small effect on FLA, with a contribution of $4.5-13.8 \%$ to the total variation (Table 3). Two QTLs, qFLA12a and qFLA12b, were located on the same chromosome (12), but at different intervals.
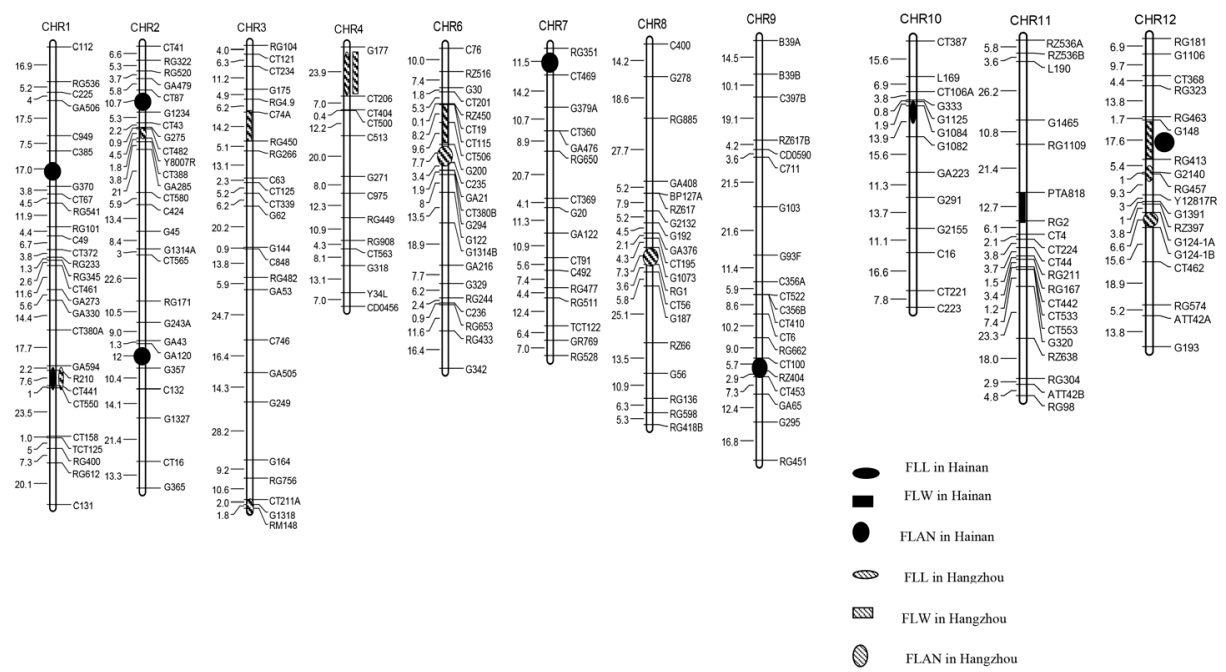

Figure 1. QTL map of flag leaf traits in ZYQ8/JX17 in different environments.
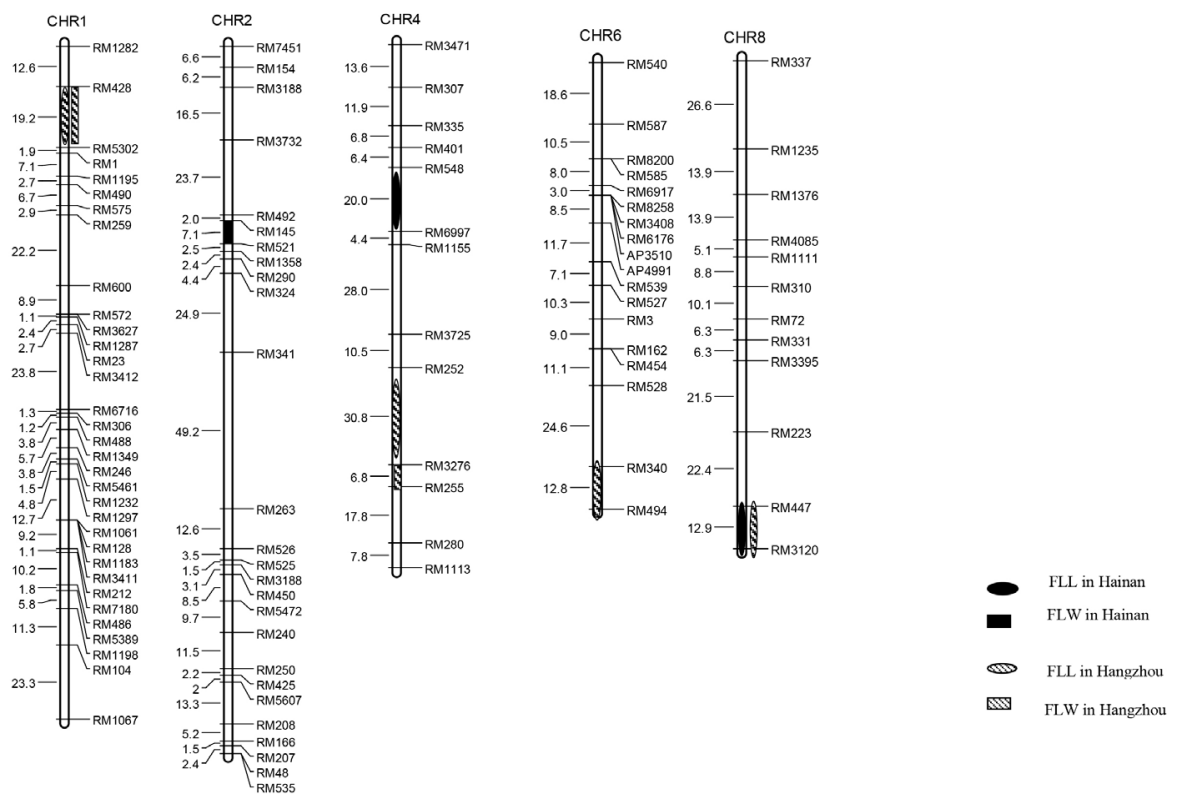

Figure 2. QTL map of flag leaf traits in CJ06/TN1 in different environments. 


\section{DISCUSSION}

In the present study, we identified a total of 30 QTLs for flag leaf traits in the $2 \mathrm{DH}$ populations. For FLL, qFLL-1a could be identified in the 2 environments in the ZYQ8/JX17 population, and qFLL-1b could be identified in the HZ environment in the CJ06/TN1 population. The qFLL-1a locus is flanked with R210 and CT441, and the restriction fragment length polymorphism marker R210 is at the physical position of $10.49 \mathrm{Mb}$ of chromosome 1 according to its sequence deposited in the Gramene website (www.gramene.org). The qFLL-1b locus is flanked by RM428 and RM5302, and RM5302 is at the physical position of approximately $4.15 \mathrm{Mb}$ on chromosome 1. Taking the phenotyping and mapping errors in the 2 populations into account, these 2 QTLs may be at the same region, and can be considered as 1 QTL. Around this region, a QTL for FLW was detected in the CJ06/TN1 population in HZ (Table 3, Figure 2). Kobayashi et al. (2003) also detected a QTL for FLL, FLW, and FLA nearby this region. Chen et al. (2012) detected a QTL for FLW at the interval of RM10116-RM220 at the physical position of 2.2 Mb. However, Li et al. (2000) and Peng et al. (2007) did not detect this QTL using the same ZYQ8/JX17 population in the Sichuan and Hubei provinces, China. Although others identified QTLs on chromosome 1, these QTLs may not be as same as that examined in this study (Wang et al., 2004; Peng et al., 2007). Three QTLs were detected for FLL on chromosome 4 in 2 populations. The qFLL-4a locus flanked by G177 and CT206 detected in the ZYQ8/JX17 population was located at approximately $22.8 \mathrm{Mb}$, and the qFLL-4b locus flanked by RM252 and RM3276 detected in the CJ06/TN1 population was at approximately $25.3 \mathrm{Mb}$, and thus, these 2 QTLs may be at the same locus. qFLL-4b is closely linked with qFLW-4b, as they shared a common marker of RM3276, and the qFLL-4c and qFLW-4b may be the same locus with pleiotropic effects on FLL and FLW. Li et al. (2000) and Peng et al. (2007) also detected qFLL-4a in an additional 2 environments in the Sichuan and Hubei Provinces. Kobayashi et al. (2003) also detected a stable QTL for FLL and FLW around this region. Chen et al. (2012) and Ding et al. (2011) detected a major QTL for FLW at the nearby region of qFLL- $4 \mathrm{~b}$, they finely mapped this QTL and delimitated it to a 38-75-kb interval where the gene NAL1 is located. Another QTL on chromosome 4 for FLL, qFLL-4c detected in the CJ06/TN1 population, was found to be far from qFLL-4b, and thus this QTL should be different from qFLL-4b. On chromosome 8, 1 QTL, qFLL-8, at the interval of RM447-RM3120, was detected in both environments in the CJ06/TN1 population. Li et al. (2000) and Peng et al. (2007) also mapped a QTL for FLL to chromosome 8 flanked by G2132 at the physical position of $19.1 \mathrm{Mb}$, while qFLL-8 was at $27.8 \mathrm{Mb}$; whether they are the same locus must be further examined. qFLL-3 and qFLL-12 were also detected by Li et al. (2000) and Peng et al. (2000).

For FLW, 4 QTLs were detected on chromosomes 1, 3, 8, and 12 in the ZYQ8/JX17 DH population by Li et al. (2000) in the Sichuan Province, in which the interval on chromosome 12 was essentially the same as that of qFLW12 (Table 3). QTLs on chromosomes 3, 4, and 6 detected in this study have been reported previously (Li et al., 2000), but because of the different populations used, it was not clear whether they were in the same locus. QTL qFLW3, located on the long arm of chromosome 3, was far from $l w 23$, located on the same chromosome using the same population reported by Li et al. (2000), and thus they were different QTLs. The QTL qFLW11 on chromosome 11 has not been reported previously, which may be a novel QTL controlling FLW. In Hangzhou and Hainan, 2 and 1 QTLs were detected by using the CJ06/TN1 DH population, respectively. QTLs qFLW1 and qFLW4b detected in Hangzhou 
were at the same regions as qFLL-1b and qFLL-4b, respectively. However, qFLW-2 detected in Hainan has not been reported previously, which may be a novel QTL controlling FLW.

Li et al. (1999) and Dong et al. (2003) detected QTLs controlling FLA on chromosomes 2, 5, 6, 7, and 9 and 1, 2, 3, and 12 using different populations in Hainan, respectively. In this study, 9 QTLs controlling FLA were detected in the ZYQ8/JX17 DH population. Six QTLs were detected in Hainan on chromosomes 1, 2, 2, 7, 9, and 12, while only 3 QTLs were detected in Hangzhou on chromosomes 6, 8, and 12. Based on the comparison of the physical position between the related markers, qFLA-1, qFLA-2a, and qFLA-7 were in the same region as the QTLs detected by Kobayashi et al. (2003), and qFLA-8 was in the same region as that detected by $\mathrm{Hu}$ et al. (2012). The two loci of qFLA-12 detected in the 2 environments were slightly different, but their $\log 10$-likelihood values and additive effect values were similar (Table 3), and thus they may be the same detected at different environments, resulting from an error, such as phenotypic measurement and statistics. Dong et al. (2003) detected this QTL at the same interval, indicating that this QTL was stably expressed. QTL qFLA-6 (CT506-G200) on chromosome 6 and qFLA-6 (C-RG424) detected by Li et al. (1999) may be in the same region and be the same QTLs.

QTLs are greatly affected by environmental conditions. Some QTLs that could be detected in different environments suggests their stable expression, while others were specifically expressed if they could only be detected at certain environments. As stated above, the QTLs on chromosomes 1, 4, and 8 for FLL and on chromosome 12 for FLA were quite stable, as they were detected either within the $2 \mathrm{DH}$ populations or in the 2 environments. Particularly, the qFLL4a was a stable QTL for FLL, with pleiotropic effects on FLW in the long-day light environment. qFLL2, qFLL3, and qFLL12 were only identified in the long-day light environment and qFLL10 in the short-day light environment in the ZYQ8/JX17 population, indicating that QTLs are greatly influenced by the environmental conditions.

Correlation analysis indicated that rice flag leaf traits were related to plant height $(\mathrm{PH})$ and heading date (HD) in this study (data not shown). We found that qFLL1a in the ZYQ8/JX17 population was in the same region as qHPH1-2 (Ma et al., 2009a) for plant height, and qFLL6 in CJ06/TN1 and qFLW6 in the ZYQ8/JX17 population were co-localized to the same loci for plant height (Ma et al., 2009b,c). In the CJ06/TN1 population, qFLL4b and qFLL4c were near qHPH-4 (or qLPH), or share the same marker (Ma et al., 2009b) for plant height. We also found that QTLs on chromosomes 2, 6, and 8 for FLL and FLW in the CJ06/TN1 population were co-located with QTLs for HD (Ma et al., 2009c). Other studies also reported high correlations between flag leaf traits and yield-related traits (Li et al., 1998; Murchie et al., 2002; Thomson et al., 2003). The significant correlations and co-location of QTLs for the correlated traits indicate a pleiotropic effect of the same gene/QTL or tight gene linkage. The 2 populations were typical crosses between indica and japonica rice. Indica rice is temperature-sensitive, while japonica rice is photosensitive. Under the long-day conditions in Hangzhou, indica and indica-inclined DH lines have shorter growth period and lower plant height, leading to smaller flag leaf length and width. The performance of japonica and japonica-inclined lines in Hangzhou is opposite to those of indica rice. Under the short-day conditions in Hainan, the performance of indica and japonica is opposite to that in Hangzhou. This may be the major environmental factor for the different results of QTL mapping between Hangzhou and Hainan. Nevertheless, QTLs detected in both Hangzhou and Hainan were unrelated to the environment.

In this study, QTLs mapping for FLL, FLW, and FLW were conducted using 2 populations in 2 environments, verifying some results of previous studies, while also revealing new 
loci. All results suggest that QTLs located on chromosomes 1, 4, and 8 are good targets for the further study of fine mapping and cloning to understand the molecular basis of leaf development. In rice breeding programs, the results indicate that appropriate plant height and heading date are indices of flag leaf traits. These QTLs are useful for marker-assisted selection for improving the plant architecture and yield potential.

\section{ACKNOWLEDGMENTS}

Research was supported by the National Natural Science Foundation of China (\#31071080), the National High Technology Research and Development Program "863" (\#2014AA10A604-1), a grant from the Zhejiang Province for Public Welfare Project (\#2011C22002).

\section{REFERENCES}

Bao JS, Kong XL, Xie JK and Xu LJ (2004). Analysis of genotypic and environmental effects on rice starch. 1. Apparent amylose content, pasting viscosity, and gel texture. J. Agric. Food Chem. 52: 6010-6016.

Cao GQ, Gao YM and Zhu J (2007). QTL analysis for flag leaf length in a rice DH population under multi-environments. Acta Agron. Sin. 33: 223-229.

Chen M, Luo J, Shao G, Wei X, et al. (2012). Fine mapping of a major QTL for flag leaf width in rice, qFLW4, which might be caused by alternative splicing of NAL1. Plant Cell Rep. 31: 863-872.

Ding X, Li X and Xiong L (2011). Evaluation of near-isogenic lines for drought resistance QTL and fine mapping of a locus affecting flag leaf width, spikelet number, and root volume in rice. Theor. Appl. Genet. 123: 815-826.

Dong GJ, Hiroshi FJ, Teng S, Hu XM, et al. (2003). QTL analysis of flag leaf angle in rice. Chin. J. Rice Sci. 17: $219-222$

Dong Y, Kamiunten H, Ogawa T, Tsuzuki E, et al. (2004). Mapping of QTLs for leaf developmental behavior in rice (Oryza sativa L.). Euphytica 138: 169-175.

Farooq M, Tagle AG, Santos RE, Ebron LA, et al. (2010). Quantitative trait loci mapping for leaf length and leaf width in rice cv. IR64 derived lines. J. Integr. Plant Biol. 52: 578-584.

Fujino K, Matsuda Y, Ozawa K, Nishimura T, et al. (2008). NARROW LEAF 7 controls leaf shape mediated by auxin in rice. Mol. Genet. Genomics 279: 499-507.

Hu J, Zhu L, Zeng D, Gao Z, et al. (2010). Identification and characterization of NARROW AND ROLLED LEAF 1, a novel gene regulating leaf morphology and plant architecture in rice. Plant Mol. Biol. 73: 283-292.

Hu WD, Zhang H, Jiang JH, Wang YY, et al. (2012). Genetic analysis and QTL mapping of large flag leaf angle trait in japonica rice. Rice Sci. 19: 277-285.

Kobayashi S, Fukuta Y, Morita S, Sato T, et al. (2003). Quantitative trait loci affecting flag leaf development in rice (Oryza sativa L.). Breed. Sci. 53: 255-262.

Li Z, Pinson SRM, Stansel JW and Paterson AH (1998). Genetic dissection of the source-sink relationship affecting fecundity and yield in rice (Oryza sativa L.) Mol. Breed. 4: 419-426.

Li ZK, Paterson AH, Pinson SRM and Stansel JW (1999). RFLP facilitated analysis of tiller and leaf angles in rice (Oryza sativa L.). Euphytica 109: 79-84.

Li SG, He P, Wang YP, Li HY, et al. (2000). Genetic analysis and gene mapping of the leaf traits in rice (Oryza saliva L.). Acta Agron. Sin. 26: 261-265.

Li SG, Ma YQ, He P, Wang YP, et al. (2002). Comparative mapping of quantitative trait loci for heading date and plant height in cultivated rice (Oryza sativa L.) across environments. Acta Agron. Sin. 28: 546-550.

Ling YH, Yang ZL, Zhong BQ, Zhao FB, et al. (2008). Genetic analysis on characters of functional leaves in rice. Chinese J. Rice 22: 45-50.

Lu YG, Zeng SX, Li ZB and Wang RH (1979). A study on the phenotypic expression and genetic transmission of dwarf gene sources of early Hsein rice in China. Acta Genet. Sin. 6: 311-321.

Lu CG, Zong SY, Zou JSi and Yao KM (2005). Leaf morphological factors and their heredity in F1 of rice. Acta Agron. Sin. 31: 1074-1079.

Ma LY, Xiao P, Cai J, Li XM, et al. (2009a). Analysis of quantitative trait loci for panicle layer uniformity in rice (Oryza sativa L.). Cereal Res. Commun. 37: 383-390. 
Ma LY, Bao JS, Guo LB, Zeng DL, et al. (2009b). Quantitative trait loci for panicle layer uniformity identified in doubled haploid lines of rice in two environments. J. Integr. Plant Biol. 51: 818-824.

Ma LY, Yang CD, Zeng DL, Cai J, et al. (2009c). Mapping QTLs for heading synchrony in a doubled haploid population of rice (Oryza sativa L.) in two environments. J. Genet. Genomics 35: 297-304.

McCouch SR, Cho YG and Yano M (1997). Report on QTL nomenclature. Rice Genet. Newsl. 14: 11-14.

Murchie EH, Yang J, Hubbart S, Horton P, et al. (2002). Are there associations between grain-filling rate and photosynthesis in the flag leaves of field-grown rice? J. Exp. Bot. 53: 2217-2224.

Pan XB, Han YB, Chen ZX and Zhang HX (2004). Progress in genetic improvement of rice plant morphological characters. J. Yangzhou Univ. 25: 36-40.

Peng MM, Yang GH, Zhang JJ, An BG, et al. (2007). QTL analysis for flag leaf morphological trait s in rice (Oryza sativa L.) under different genetic backgrounds. Chin. J. Rice Sci. 21: 247-252.

Qi J, Qian Q, Bu Q, Li S, et al. (2008). Mutation of the rice Narrow leaf1 gene, which encodes a novel protein, affects vein patterning and polar auxin transport. Plant Physiol. 147: 1947-1959.

Shen B, Zhuang JY, Zhang KQ, Xia QQ, et al. (2003). QTLs mapping of leaf traits and root vitality in a recombinant inbred line population of rice. J. Genet. Genomics 30: 1133-1139.

Shen FC (1983). Several opinions on the utilization of rolled leaf character in rice breeding. Guizhou Agric. Sci. 6-8.

Sonah H, Deshmukh R, Chand S, Srinivasprasad M, et al. (2012). Molecular mapping of quantitative trait loci for flag leaf length and other agronomic traits in rice (Oryza sativa). Cereal Res. Commun. 40: 362-372.

Tang JJ (1998). Study on the contribution of assimilates to grain yield formation of high-yielding intergenic hybrid (Oryza $\times$ Sorghum) among canopy leaves. J. Zhejiang Univ. 32: 116-121.

Thomson MJ, Tai TH, McClung AM, Lai XH, et al. (2003). Mapping quantitative trait loci for yield, yield components and morphological traits in an advanced backcross population between Oryza rufipogon and the Oryza sativa cultivar Jefferson. Theor. Appl. Genet. 107: 479-493.

Tong HH, Mei HW, Xing YZ, Cao YP, et al. (2007). QTL analysis for morphological and physiological characteristics of flag leaf at the late developmental stage in rice. Chin. J. Rice Sci. 21: 493-499.

Wang YP, Zeng JP, Guo LB, Xing YZ, et al. (2004). QTL and correlation analysis on characters of top three leaves and panicle weight in rice (Oryza sativa). Chin. J. Rice Sci. 19: 13-20.

Wang P, Zhou G, Yu H and Yu S (2011). Fine mapping a major QTL for flag leaf size and yield-related traits in rice. Theor. Appl. Genet. 123: 1319-1330.

Yan YM and Wang XX (1990). Genetic analysis of flag leaf shape between indica/japonica crosses in rice. Hereditas 12: $1-4$.

Yang CD, Yuan PR, Zhou N, Zhu DF, et al. (2001). Analysis on relationship between characters of leaf type and yield components. Chin. J. Rice Sci. 15: 70-72.

Yue B, Xue WY, Luo LJ and Xing YZ. (2006). QTL analysis for flag leaf characteristics and their relationships with yield and yield traits in rice. Yi Chuan Xue Bao 33: 824-832.

Zhang GH, Zeng DL, Hu SK, Su Y, et al. (2006). QTL analysis of traits concerned submergence tolerance at seedling stage in rice (Oryza sativa L.). Acta Agron. Sin. 32: 1280-1286.

Zhang YJ and Wang HB (2001). The effect of leaf numbers and quality to grain setting ability after heading in rice. Till. Cultiv. 22-24. 